\title{
Effect of ventricular myocardium characteristics on the defibrillation threshold
}

\author{
Li Qian $^{\mathrm{a}}$, Jianfei Wang ${ }^{\mathrm{a}}$, Lian Jin ${ }^{\mathrm{a}}$, Biao Song ${ }^{\mathrm{a}}$ and Xiaomei $\mathrm{Wu}^{\mathrm{a}, \mathrm{b}, \mathrm{c}, *}$ \\ ${ }^{a}$ Electrical Engineering Department, Fudan University, Shanghai, China \\ ${ }^{\mathrm{b}}$ Shanghai Engineering Research Center of Assistive Devices, The Key Laboratory of Medical Imaging \\ Computing, Shanghai, China \\ ${ }^{\mathrm{c}}$ Computer Assisted Intervention (MICCAI) of Shanghai, Shanghai, China
}

\begin{abstract}
Myocardium characteristics differ markedly among individuals and play an important role in defibrillation threshold. The accuracy of simulation models used in most published studies are still have room to be improved and most of them only discussed the effect of myocardial anisotropy on defibrillation threshold. In our manuscript, a rabbit ventricular finiteelement (FE) volume conductor model with high precision was constructed. Ventricular myocardium characteristics include cardiomyocyte coupling and the degree of myocardial anisotropy, which are represented as the value and the ratio of anisotropic conductivity, respectively. Quantitative analysis was performed simultaneously in terms of cardiomyocyte coupling and the degree of myocardial anisotropy. Based on this, the combined effects of these two factors were further discussed. The electric field distributions of shocks and the defibrillation thresholds under different myocardial characteristics were simulated on this model. The simulation results revealed that as the degree of myocardial anisotropy increases, defibrillation threshold increases, and cardiomyocyte decoupling (decrease in electrical conductivity) can considerably increase the defibrillation threshold.
\end{abstract}

Keywords: Ventricular myocardium characteristics, defibrillation threshold (DFT), finite-element (FE) volume conductor model, cardiomyocyte coupling, myocardial anisotropy

\section{Background and objective}

In China, more than 500,000 people die each year from sudden cardiac death (SCD) [1]; worldwide, this figure has reached an alarming 7 million [2]. One of the important causes of SCD is ventricular fibrillation, and the only effective clinical way to terminate it is by shock defibrillation. Myocardial characteristics play an important role in shock defibrillation effect, which differs markedly under different pathological or physiological conditions of different individuals. For instance, cardiomyocyte coupling will decrease with age; myocardial ischemia can lead to cardiomyocyte decoupling at the ischemic area; the degree of myocardial anisotropy increases for the patients with cardiac hypertrophy. Therefore, it is instructive to understand the effect of ventricular myocardium characteristics on the defibrillation threshold (DFT). A wrong assessment of DFT will result in a failed shock defibrillation [3]. Therefore, studying the effect of myocardium characteristics on defibrillation has not only important theoretical significance, but also important clinical value.

\footnotetext{
${ }^{*}$ Corresponding author: Xiaomei Wu, Electrical Engineering Department, Fudan University, Shanghai 200433, China. Tel.: +862165643709 081; E-mail: xiaomeiwu@ fudan.edu.cn.
}

0928-7329/18/\$35.00 (c) 2018 - IOS Press and the authors. All rights reserved

This article is published online with Open Access and distributed under the terms of the Creative Commons Attribution NonCommercial License (CC BY-NC 4.0). 
The distribution of electric field in the heart is closely related to the defibrillation results [4]. It is almost impossible to measure the distribution of electric field in the myocardium in vivo, so finiteelement (FE) modeling has been widely used in such studies. During the previous three decades, various three-dimensional cardiac models have been established. Among them, volume conductor models can best reflect the electric field distribution in ventricles. Some examples include the human thoracic model constructed by Camacho et al. [5], the dog chest model developed by Karlon et al. [6], and the human thoracic model built by Kinst et al. [7]. Besides electric field, these models used current density as an indicator of defibrillation effects, which aimed to finding the relationship between current density and DFT. In 1975, the embryonic form of the critical mass hypothesis was proposed [8] and in the midnineties, this method for calculating the DFT has been generally accepted [9]. Using this hypothesis, many models were established to study the effect of defibrillation field on DFT, such as the porcine thoracic model constructed by Wang et al. [10] and the dog heart model built by Yang et al. [11]. With the understanding of the influence of myocardial anisotropy on the distribution of defibrillation electric field [12,13], several cardiac volume conductor models have reported factors influencing the myocardial fibers. Such as the human torso models constructed by Eason et al. [13], Aguel et al. [14], Wang et al. [15] and Modre et al. [16], and the swine chest models constructed by Panescu et al. [17].

The aforementioned studies were committed to optimizing the electrode configuration $[5-7,10,11]$ or researching the effects of myocardial anisotropy on DFT based on one group of anisotropic conductivities, which did not further discuss why fiber anisotropy could have this kind of effect on DFT [13-17].

To investigate the effect of myocardial characteristics on DFT, this manuscript used a rabbit ventricle as the anatomical model, which has higher resolution than aforementioned models [5-7,10,11,14-18]. Rabbit heart has much smaller size compared to human heart, so using this model can evidently reduce the computational complexity, significantly shortening the simulation time. In addition, the structure and electrophysiological characteristics of the human and rabbit heart have a high degree of similarity, making this model reliable [19].

In this manuscript, the DFTs in different ventricular myocardium characteristics were given in the RESULTS section. The effects of cardiomyocyte coupling, the degree of myocardial anisotropy and the combined effects of these two factors on DFT were discussed in DISCUSSION section. Model development, model parameter selection and simulation methods were discussed in METHODS section. This study provided a basis for the modeling and parameter selection of myocardial conductivity in different conditions, and thus provides some guidance for defibrillation treatment.

\section{Methods}

In this manuscript, a rabbit ventricular FE model with a resolution of approximately $250 \mu \mathrm{m}$ was constructed using the magnetic resonance imaging (MRI) data of the rabbit ventricles provided by Oxford University [20,21]. The model is $2.37 \times 2.16 \times 2.94 \mathrm{~cm}$ and contains 955,292 nodes and 50,28,264 elements. In this model, conductivity value was used to represent the cardiomyocyte coupling, and the ratio of anisotropic conductivity was used to represent the degree of myocardial anisotropy. By changing the value of the anisotropic conductivity, we studied the effect of myocardium characteristics on the DFTs based on the critical mass hypothesis. The criterion used in this study was a voltage gradient of $5 \mathrm{~V} / \mathrm{cm}$ generated over $95 \%$ of the myocardium [22].

To simplify the problem, all simulations in this manuscript used the implantable cardioverter defibrillator (ICD) electrode configuration: the anode (a thin cylindrical electrode with a height of $16 \mathrm{~mm}$ and a radius of $0.5 \mathrm{~mm}$ ) was inserted into the right ventricular cavity and the cathode (a circular patch 

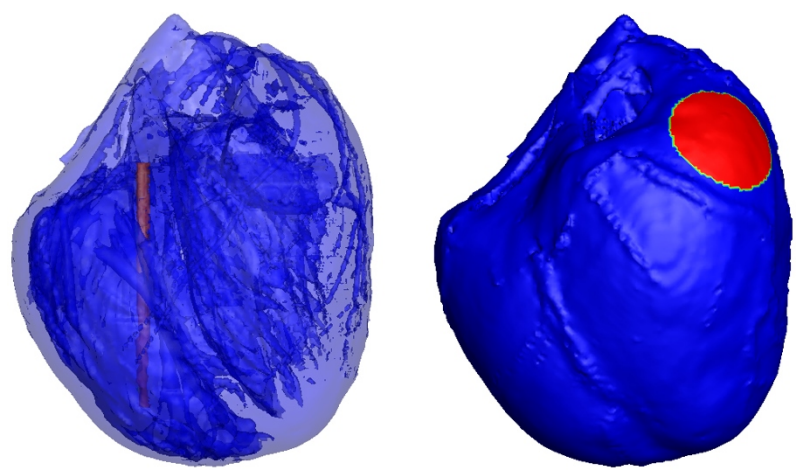

Fig. 1. ICD electrode configuration was used in the simulation. The red part of in the right ventricle as shown in left picture is the columnar electrode, the red part of on left ventricular epicardium as shown in right picture is the circular patch electrodes.

electrode with $4 \mathrm{~mm}$ radius) was placed on the upper part of the left ventricular epicardium (Fig. 1). A defibrillation threshold voltage was applied between the cylindrical electrode (anode) and the circular patch electrode (cathode) such that the critical mass hypothesis was met. In this study, the DFTs for isotropic, anisotropic, and different anisotropic conductivities were simulated.

The electric shock defibrillation problem is equivalent to the constant electric field problem in the volume conductor model. The potential in the volume conductor satisfies the Laplace equation:

$$
\nabla \cdot(\sigma \nabla \Phi)=0
$$

where $\sigma$ is the conductivity tensor, and $\Phi$ is the potential. Equation (1) is solved subject to the Dirichlet condition Eq. (2) for the anode and cathode regions, and the Neumann boundary condition for the residual ventricular surface Eq. (3).

$$
\begin{aligned}
\left.\Phi(x, y, z)\right|_{S I_{i}} & =V_{i} \\
\left.\frac{\partial \Phi}{\partial n}\right|_{S 2} & =0
\end{aligned}
$$

Equation (2) represents the Dirichlet boundary condition, which defines the myocardial surface $\left(S I_{i}\right)$ in contact with the electrode $\left(i_{\text {th }}\right)$ with a potential $V_{i}$, and the potential of $S I_{i}$ is $V_{i}$. Equation (3) represents the Neumann boundary condition, which defines the myocardial surface that is not in contact with the electrode; the derivative of the potential gradient on the surface of the myocardium is zero. The finite element method can be used to solve Eq. (1) [5] because it is a numerical simulation technique suitable for solving partial differential equations in an irregular geometric region.

The fiber conductivity tensor $\sigma$ of every element in FE model was calculated using following formula [21]:

$$
\sigma=\sigma_{t} I+\left(\sigma_{l}-\sigma_{t}\right) A A^{T}
$$

where the coefficient $\sigma_{l}$ is the conductivity along the direction of the myocardial fibers, and the coefficient $\sigma_{t}$ is the conductivity perpendicular to the myocardial fiber. $I$ is the unit matrix. $A$, the direction cosine of every element, can be obtained by the rule-based approach [23], which determines the direction of myocardial fibers. Therefore, this model reflects the fiber orientation in ventricular epicedial, myocardial, and endocardial layers.

The FE simulation software, Comsol Multiphysics 5.2 (COMSOL Inc.), was used. For an isotropic cardiac volume model, the electrical conductivity of myocardial fibers was set to $\sigma_{m}=0.25 \mathrm{~S} / \mathrm{m}$ (first 
Table 1

Electrical conductivity of myocardial fibers given by different literatures

\begin{tabular}{|c|c|c|c|c|c|c|c|}
\hline $\begin{array}{l}\text { Cited } \\
\text { paper }\end{array}$ & The model used & $\begin{array}{l}\text { Anisotropic } \\
\text { ratio }\end{array}$ & $\begin{array}{l}\text { Myocardial anisotropic } \\
\text { conductivity }(\mathrm{S} / \mathrm{m})\end{array}$ & $\bar{\sigma}$ & $\begin{array}{l}\text { Blood conductivity } \\
(\mathrm{S} / \mathrm{m})\end{array}$ & $\begin{array}{c}\text { DFT } \\
(\mathrm{V})\end{array}$ & $\begin{array}{l}\text { POD } \\
(\%)\end{array}$ \\
\hline [5] & del of human & $1: 1$ & $\sigma_{l}=\sigma_{t}=0.25$ & 0.25 & 0.8 & 113 & 0 \\
\hline [15] & Chest model of swine & $2: 1$ & $3367 ; \sigma_{t}=0.1684$ & 0.2526 & 0.649 & 122 & 7.96 \\
\hline [16] & Ventricular model of human & $4: 1$ & $\sigma_{l}=0.6 ; \sigma_{t}=0.15$ & 0.375 & 0.6 & 116 & 2.65 \\
\hline [14] & Torso model of human & $3.74: 1$ & $\sigma_{l}=0.625 ; \sigma_{t}=0.167$ & 0.396 & 0.775 & 113 & 0 \\
\hline [13] & Torso model of human & 2.648:1 & $\sigma_{l}=0.625 ; \sigma_{t}=0.236$ & 0.4305 & 0.775 & 106 & -6.19 \\
\hline [17] & Chest model of human & $2: 1$ & $\sigma_{l}=0.5 ; \sigma_{t}=0.25$ & 0.375 & 0.667 & 107 & -5.31 \\
\hline
\end{tabular}

$\sigma_{l}=$ conductivity along the direction of myocardial fiber, $\sigma_{t}=$ conductivity perpendicular to the direction of myocardial fiber, $\bar{\sigma}=$ average value of $\sigma_{l}$ and $\sigma_{t}$, DFT $=$ defibrillation voltage thresholds, POD $=$ Percentage of difference between the DFT of isotropic conductivity and the DFT of anisotropic conductivity. The units of $\sigma_{l}, \sigma_{t}$ and $\bar{\sigma}$ are $\mathrm{S} / \mathrm{m}$. The information obtained directly from different literatures is $1-4,6$ columns of the table. DFT is obtained by simulation.
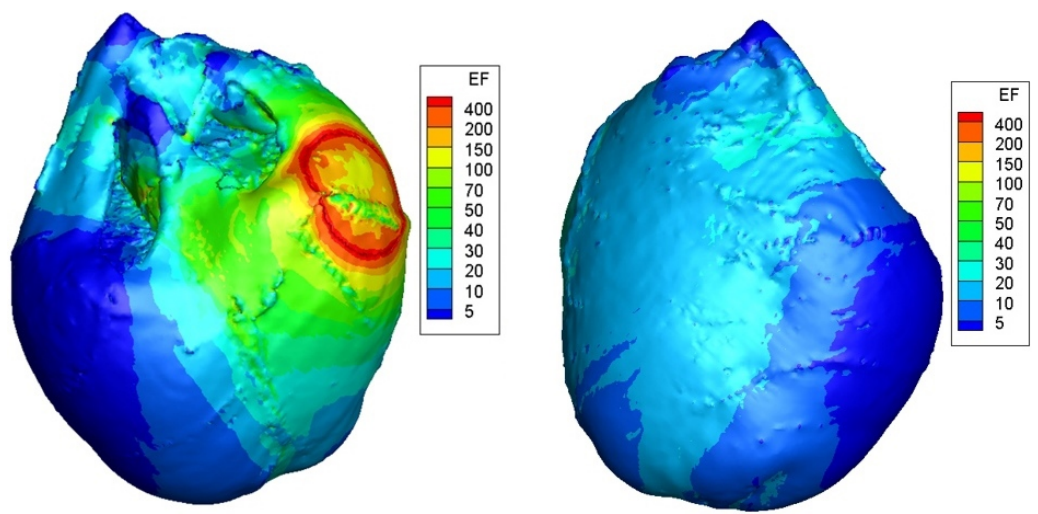

Fig. 2. Simulation result of the distribution of ventricular electric field. The red part represents the high electric field area and the blue part represents the low electric field area. The unit of electrical field (EF) is V/cm.

row, Table 1). Ventricular blood perfusion greatly changes the distribution of the electric field in the volume conductor model, which has a great impact on the DFT, so the blood perfusion was added into the ventricle. Since blood conductivity is isotropic, for all ventricular models constructed in this study, the conductivity of the intraventricular blood was set to $\sigma_{b}=0.7 \mathrm{~S} / \mathrm{m}$ (the average of the blood conductivities in Table 1).

\section{Results}

Electrical conductivity of myocardial fibers varies in different literatures. For all myocardial conductivities shown in Table 1, the corresponding distributions of electric field in the ventricle were gotten by our computer simulation (Fig. 2). The DFT (minimum defibrillation voltage that meets the critical mass hypothesis) is showed in seventh column of Table 1. Using Eq. (4), we calculated all the percentages of difference (POD, eighth column, Table 1) between the DFT of isotropic conductivity ( $\mathrm{DFT}_{\text {Iso }}$ ) and the DFT of anisotropic conductivity $\left(\mathrm{DFT}_{\mathrm{Ani}}\right)$.

$$
\mathrm{POD}=\frac{\mathrm{DFT}_{\text {Ani }}-\mathrm{DFT}_{\text {Iso }}}{\mathrm{DFT}_{\text {Iso }}} \times 100 \%
$$

The values of anisotropic conductivites were changed depending on the possible cell coupling conditions: 1 . because the cells are not prone to over-coupling, the $\sigma_{l}$ and $\sigma_{t}$ values were $<0.7 \mathrm{~S} / \mathrm{m}$ (all 


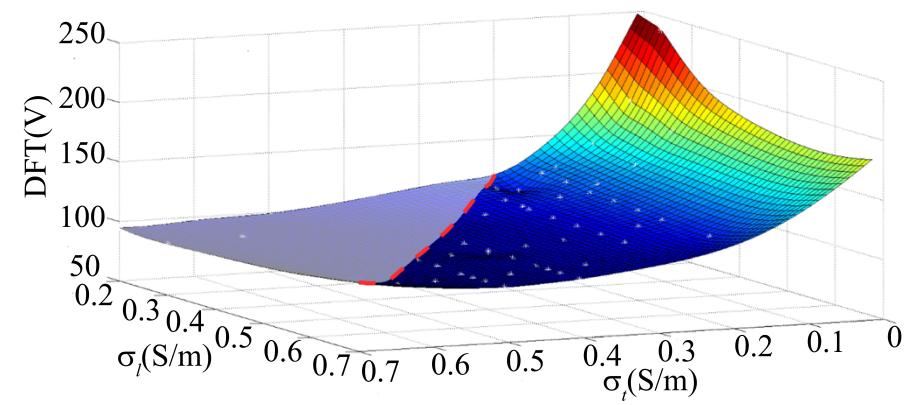

Fig. 3. Effect of cell coupling degree on defibrillation electric field distribution. As shown, the two axes of the horizontal plane respectively represent electrical conductivity along the myocardial fiber $\sigma_{l}$ and perpendicular to the myocardial fiber $\sigma_{t}$, and the axis perpendicular to the horizontal plane represents the DFT. The white dots distributed over the curved surface represent the simulated DFT. As the myocardial fiber conductivity reflects the cell coupling, follow the two restrictions mentioned above, the right side of the red dotted line is the actual possible DFTs distribution.

myocardial conductivities given in literature [5,13-17] are below $0.7 \mathrm{~S} / \mathrm{m}) ; 2$. usually the number of gap junctions along the cell fibers is more than those perpendicular to the cell fibers; therefore, $\sigma_{l} \geqslant \sigma_{t}$. Based on these two principles, the DFTs were obtained by simulation, resulting in a curved surface (Fig. 3). The effect of the anisotropy on the DFT is further explained in Fig. 4, which showed the percentage of ventricular myocardial mass below discrete potential gradients in different myocardial coupling (conductivity).

In the next part, three aspects of the problem will be discussed: 1 . The effect of intercellular coupling on the DFT, which was represented as the value of the conductivity in volume conductor model (Section 3.1); 2. The effect of the degree of the myocardial anisotropy on the DFT, which was represented as the ratio of anisotropic conductivity in volume conductor model (Section 3.2); 3. The combined effect of the above two aspects on the DFT (Section 3.3).

\subsection{Effects of intercellular coupling of cardiomyocytes on the defibrillation thresholds}

Studies have shown that cardiomyocyte coupling directly affects the degree of dispersion in myocardial repolarization and provides a major basis for reentrant arrhythmias [24]. This section investigated the effects of the degree of cardiomyocyte coupling on the DFT. By comparing the DFTs when $\bar{\sigma}=$ $0.2526 \mathrm{~S} / \mathrm{m}$ (second row, Table 1) and $\bar{\sigma}=0.375 \mathrm{~S} / \mathrm{m}$ (sixth row, Table 1), whose ratios of anisotropic conductivity are both 2:1, it was found that the DFTs are $122 \mathrm{~V}$ and $107 \mathrm{~V}$ respectively. Therefore, the tighter the intercellular coupling is, the greater the myocardium conductivity which results in the lower DFT. This is because when the electrical conductivity of the myocardium is lower, more current flows through the blood, reducing the electric field strength in the myocardium. As a result, to satisfy the critical mass hypothesis, a higher defibrillation voltage must be applied for successful defibrillation.

\subsection{Effects of myocardial anisotropic conductivity on the defibrillation thresholds}

The shape of the myocardial cell is fusiform, which has long axis and short axis. The gap junctions between the long axis of the cells connection are more intensive, and thus the electrical conductivity is higher; gap junctions between the short axes of the cells are rare, and thus the electric conductivity is lower $\left(\sigma_{l}>\sigma_{t}\right)$. So the intercellular coupling can be described by anisotropic conductivity. In addition, the distribution of gap junctions depends on the age of species and calcium ion concentration of the cell [24]. Therefore, the anisotropic conductivities vary widely in different studies (Table 1). 


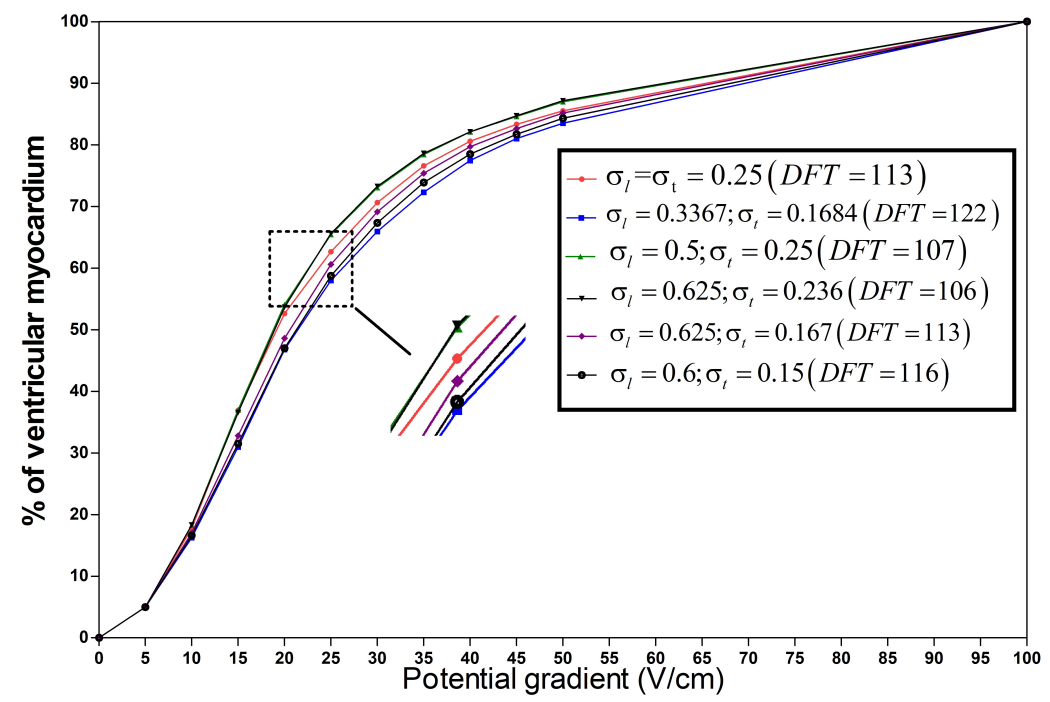

Fig. 4. The percentage of ventricular myocardial mass below discrete potential gradients in different anisotropic conductivity. The right diagram shows the case in Table 1 with different anisotropy $\operatorname{ratios}\left(\sigma_{l} / \sigma_{t}\right)$, which are 1:1, 2:1, 2:1, 2.648:1, 3.74:1, 4:1 respectively. The units of conductivity $\sigma_{l}$ and $\sigma_{t}$ are $\mathrm{S} / \mathrm{m}$, the units of DFTs are V.

To investigate the effect of anisotropic conductivity on DFT, the DFTs of the isotropic conductivity (first row, Table 1) and anisotropic conductivity with 2:1 anisotropic ratio (second row, Table 1) were compared. The $\bar{\sigma}$ of these two sets of myocardial conductivities are both $0.25 \mathrm{~S} / \mathrm{m}$. The simulated DFTs of the isotropic and anisotropic myocardial fibers were $\mathrm{DFT}_{\text {Iso }}=113 \mathrm{~V}$ and $\mathrm{DFT}_{\mathrm{Ani}}=122 \mathrm{~V}$, respectively. The POD between them was 7.96\% calculated by using Equation (5).

Furthermore, both the conductivity of $4: 1$ anisotropic ratio (third row, Table 1 ) and the conductivity of 2:1 anisotropic ratio (sixth row, Table 1) have $\bar{\sigma}$ value of $0.375 \mathrm{~S} / \mathrm{m}$. However, the DFTs are $116 \mathrm{~V}$ and $107 \mathrm{~V}$, so the percentages of difference are $2.65 \%$ and $-5.31 \%$ respectively. It can be seen that the degree of anisotropy has a significant effect on the DFT, the higher the degree of anisotropy, the higher the DFT.

\subsection{The effect of cardiomyocyte coupling and the degree of anisotropy on defibrillation thresholds}

Previous studies in literature [8] have already provided that myocardial fibers have substantial electrical anisotropy, and myocardial anisotropy affects the distribution of voltage and defibrillation electric field. James Eason et al. compared the DFTs of the isotropic conductivity $\left(\sigma_{l}=\sigma_{t}=0.28 \mathrm{~S} / \mathrm{m}\right)$ and the anisotropic conductivity $\left(\sigma_{l}=0.625 \mathrm{~S} / \mathrm{m}, \sigma_{t}=0.236 \mathrm{~S} / \mathrm{m}, \bar{\sigma}=0.43 \mathrm{~S} / \mathrm{m}, \sigma_{l} / \sigma_{t}=2.6: 1\right)$ and obtained the conclusion that the distribution of the potential gradient depends primarily on the electrode position (considered a first-order influence), and the direction of a myocardial fiber only plays a modulatory role (considered a second-order influence) [13]. The above study did not take the value of myocardial conductivity into account when discussing the effect of anisotropic conductivity on DFT, so in this section, the effects of cardiomyocyte coupling and the degree of anisotropy on DFT were both investigated. As shown in Fig. 3, when $\sigma_{t}$ is fixed, DFTs only decrease slightly with increasing $\sigma_{l}$. This is the result of increasing myocardial conductivity and the increasing degree of anisotropy counteracting each other to some extent; when fixing $\sigma_{l}$, DFTs significantly decrease with increasing $\sigma_{t}$ because the increasing myocardial conductivity and the decreasing degree of anisotropy are overlapping to some extent. These 
findings demonstrated the effects of myocardial electrical conductivity and the degree of anisotropy on DFTs.

As shown in Fig. 4, with the increasing proportion of the low-intensity region, the curves shifted left, indicating a smaller or constant DFT. The DFTs of the red line (isotropic conductivity, $\sigma_{l}=\sigma_{t}=$ $0.25 \mathrm{~S} / \mathrm{m}$ ) and violet line (anisotropic conductivity; $\sigma_{l}=0.625 \mathrm{~S} / \mathrm{m} ; \sigma_{t} 0.167 \mathrm{~S} / \mathrm{m}$ ) were $113 \mathrm{~V}$, but the average conductivity value of the anisotropic myocardium $(0.396 \mathrm{~S} / \mathrm{m})$ was much higher than that of the isotropic myocardium, and the proportion of the ventricular electric field distribution in the lowintensity region was higher under isotropic conditions. Therefore, the degree of myocardial anisotropy had an effect on defibrillation electric field distribution. When conductivity is closer to isotropy $\left(\sigma_{l} / \sigma_{t}\right.$ closer to 1), the proportion of the defibrillation electric field to the low-intensity region is higher, and the DFT is lower.

\section{Conclusions}

This study focused on the effects of ventricular myocardium characteristics on DFT. It is concluded that the DFTs and electrical conductivity of myocardial fibers are closely related. Higher electrical conductivities correlates with lower DFTs. In addition, the DFTs are affected by the degree of myocardial anisotropy. Higher degree of myocardial anisotropy correlates with higher DFTs.

Cardiac modeling and simulation are important techniques for evaluating defibrillation electric field distribution; the accuracy and complexity of the model directly affect the simulation results and efficiency. This study not only provides a basis for cardiac modeling and simulation of defibrillation electric field distribution, but also provides a new insight into the differences in the DFTs of clinical patients.

\section{Acknowledgments}

The authors would like to thank the Department of Computer Science, Oxford University for providing the data of rabbit ventricular model. This work received financial support from Science and Technology Commission of Shanghai (Grant No. 16441907900).

\section{Conflict of interest}

None to report.

\section{References}

[1] Hua W, Zhang L, Wu Y, et al. Incidence of sudden cardiac death in china analysis of 4 regional populations. Journal of the American College of Cardiology 2009; 54(12): 1110-1118.

[2] Smith TW, Cain ME. Sudden cardiac death: Epidemiologic and financial worldwide perspective. Journal of Interventional Cardiac Electrophysiology 2007; 17(3): 199-203.

[3] Li Y, Ristagno G, Yu T, et al. A comparison of defibrillation efficacy between different impedance compensation techniques in high impedance porcine model. Resuscitation 2009; 80(11): 1312-1317.

[4] Jianfei W, Lian J, Xiaomei W, et al. Low-energy defibrillation research using a rabbit ventricular model: Optimizing the potential gradient distribution using multiple epicardial electrodes. Conference Proceedings Annual International Conference of the IEEE Engineering in Medicine and Biology Society. IEEE Engineering in Medicine and Biology Society. Annual Conference 2016; 2753-2756. 
[5] Camacho MA, Lehr JL, Eisenberg SR. A 3-dimensional finite-element model of human transthoracic defibrillationpaddle placement and size. IEEE Transactions on Biomedical Engineering 1995; 42(6): 572-578.

[6] Karlon WJ, Eisenberg SR, Lehr JL. Effects of paddle placement and size on defibrillation current distribution - a 3dimensional finite-element model. IEEE Transactions on Biomedical Engineering 1993; 40(3): 246-255.

[7] Kinst TF, Sweeney MO, Lehr JL, et al. Simulated internal defibrillation in humans using an anatomically realistic threedimensional finite element model of the thorax. Journal of Cardiovascular Electrophysiology 1997; 8(5): 537-547.

[8] Zipes DP, Fischer J, King RM, et al. Termination of ventricular-fibrillation in dogs by depolarizing a critical amount of myocardium. American Journal of Cardiology 1975; 36(1): 37-44.

[9] Aguel F, Eason JC, Trayanova NA, et al. Impact of transvenous lead position on active-can ICD defibrillation: A computer simulation study. Pace-Pacing and Clinical Electrophysiology 1999; 22(12): 158-164.

[10] Wang YQ, Schimpf PH, Haynor DR, et al. Analysis of defibrillation efficacy from myocardial voltage gradients with finite element modeling. IEEE Transactions on Biomedical Engineering 1999; 46(9): 1025-1036.

[11] Yang F, Sha Q, Patterson RP. A novel electrode placement strategy for low-energy internal cardioversion of atrial fibrillation: A simulation study. International Journal of Cardiology 2012; 158(1): 149-152.

[12] Plonsey R, Barr R. The 4-electrode resistivity technique as applied to cardiac-muscle. IEEE Transactions on Biomedical Engineering 1982; 29(7): 541-546.

[13] Eason J, Schmidt J, Dabasinskas A, et al. Influence of anisotropy on local and global measures of potential gradient in computer models of defibrillation. Annals of Biomedical Engineering 1998; 26(5): 840-849.

[14] Aguel F, Eason JC, Trayanova NA, et al. Impact of transvenous lead position on active-can ICD defibrillation: A computer simulation study. Pace-Pacing and Clinical Electrophysiology 1999; 22(12): 158-164.

[15] Wang YQ, Haynor DR, Kim Y. An investigation of the importance of myocardial anisotropy in finite-element modeling of the heart: Methodology and application to the estimation of defibrillation efficacy. IEEE Transactions on Biomedical Engineering 2001; 48(12): 1377-1389.

[16] Modre R, Seger M, Fischer G, et al. Cardiac anisotropy: Is it negligible regarding noninvasive activation time imaging? IEEE Transactions on Biomedical Engineering 2006; 53(4): 569-580.

[17] Panescu D, Webster JG, Tompkins WJ, et al. Optimization of cardiac defibrillation by 3-dimensional finite-element modeling of the human thorax. IEEE Transactions on Biomedical Engineering 1995; 42(2): 185-192.

[18] Eason J, Schmidt J, Dabasinskas A, et al. Influence of anisotropy on local and global measures of potential gradient in computer models of defibrillation. Annals of Biomedical Engineering 1998; 26(5): 840-849.

[19] Arevalo HJ, Boyle PM, Trayanova NA. Computational rabbit models to investigate the initiation, perpetuation, and termination of ventricular arrhythmia. Progress in Biophysics and Molecular Biology 2016; 121(2): 185-194.

[20] Bordas R, Gillow K, Lou Q, et al. Rabbit-specific ventricular model of cardiac electrophysiological function including specialized conduction system. Progress in Biophysics and Molecular Biology 2011; 107(1): 90-100.

[21] Clayton RH, Panfilov AV. A guide to modelling cardiac electrical activity in anatomically detailed ventricles. Progress in Biophysics and Molecular Biology 2008; 96(1-3): 19-43.

[22] Jolley M, Stinstra J, Pieper S, et al. A computer modeling tool for comparing novel ICD electrode orientations in children and adults. Heart Rhythm 2008; 5(4): 565-572.

[23] Bishop MJ, Plank G, Burton RAB, et al. Development of an anatomically detailed MRI-derived rabbit ventricular model and assessment of its impact on simulations of electrophysiological function. AJP: Heart and Circulatory Physiology 2010; 298(2): H699-H718.

[24] Spach MS. Changes in the topology of gap-junctions as an adaptive structural response of the myocardium. Circulation 1994; 90(2): 1103-1106. 